\title{
Research of the Influence of Nonlinear Primary Magnetization Curves of Magnetic Circuits of Electromagnetic Transducers of the Three-phases Current
}

\author{
Siddikov Ilkhomjon Khakimovich*, Sattarov Khurshid Abdishukurovich, \\ Khujamatov Khalimjon Ergashevich \\ “Power Supply Systems”, Tashkent University of Information Technology, Uzbekistan
}

Copyright $\mathrm{O} 2016$ by authors, all rights reserved. Authors agree that this article remains permanently open access under the terms of the Creative Commons Attribution License 4.0 International License

\begin{abstract}
During monitoring and controlling of the processes of transmission and distribution electricity and power from three-phases nets are important accuracy and commonality primary transducer elements and devices. Incorrect operations and improper signals about conversion of primary values and parameters of the energy and power three-phases current associated with losses indicators of energy and resource in electrical power systems.
\end{abstract}

Keywords Magnetic Flux, Electric, Magnetic Circuits

\section{Introduction}

Classical single-phase current transformers using currently in electrical nets for monitoring and control values and parameters of the reactive component of electric energy and power, do not take for account of mutual influence of magnetic flux fields generated by currents of three-phases power supply systems. They do not provide necessary accuracy, especially when three-phases primary current have unbalance, do not have sufficient community, covering only sizes and parameters of the electric and magnetic circuits, because distribution of the magnetic signal conversion systems have nonlinearity and heterogeneous distributed parameters [1].

Research dates of the classic single-phase primary electromagnetic transducers not provided enough accurate and simultaneous information on the values and parameters of reactive electric energy and power of three-phases nets. On the determining above, become necessary to develop a new electromagnetic signal transducer of the value of reactive power of electricity, to account the features of the current of three-phases nets, which differ from each others in magnitudes and phases [2].

\section{Methodology \& Methods}

In fig. 1 shown equivalent circuit of magnetic circuit of the electromagnetic transducers of three-phase current, which formed for account the assumption of the magnetic permeability of steel, when entire range of induction is constant $(\mu=$ const $)$. Flux, closes at the outer surface of the outer cylindrical magnetic core, can be neglected.

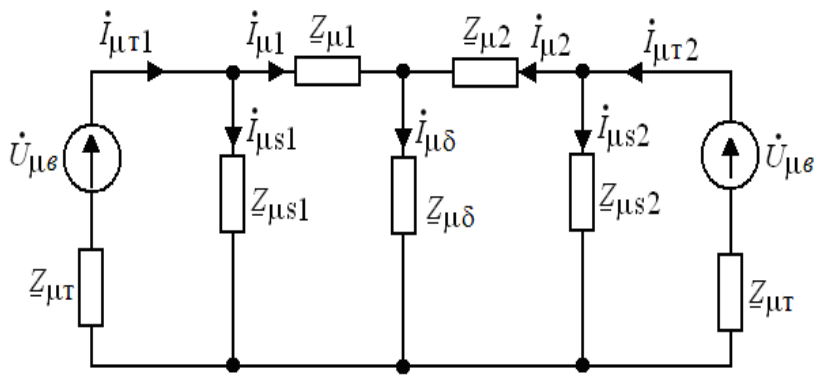

Figure 1. The equivalent circuit of the magnetic circuit of the electromagnetic transducers of three-phases current

For simplify the calculation of magnetic circuit of the electromagnetic transducer of three-phases currents used parametric structural diagrams (PSD). According fig. 1, for the construction of PSD determined following designations:

$$
\begin{gathered}
\underline{Z}_{\mu \mathrm{r}}=R_{\mu \mathrm{r}}+j\left(\omega L_{\mu \mathrm{r}}-\frac{1}{\omega C_{\mu \mathrm{r}}}\right), \\
\underline{Z}_{\mu 1}=R_{\mu 1}+j\left(\omega L_{\mu 1}-\frac{1}{\omega C_{\mu 1}}\right),
\end{gathered}
$$




$$
\underline{Z}_{\mu 2}=R_{\mu 2}+j\left(\omega L_{\mu 1}-\frac{1}{\omega C_{\mu 1}}\right) \text { - full complex }
$$

resistance of magnetic portions;

$$
\underline{Z}_{\mu S 1}=-j \frac{1}{\omega C_{\mu S 1}}, \underline{Z}_{\mu S 2}=-j \frac{1}{\omega C_{\mu S 2}}
$$

complex reactive magnetic resistance of the air clearance;

$$
\underline{Z}_{\mu \delta}=\underline{Z}_{\mu c}+\underline{Z}_{\mu g}-\text { a general resistance fully }
$$

integrated in the flow path is closed through the inertial element;

$$
\underline{Z}_{\mu c}, \underline{Z}_{\mu g} \quad \text { - full complex resistance respectively }
$$
movable core - the inertial element and working backlashes;

$R_{\mu 1}, L_{\mu 1}, C_{\mu 1}$ and $R_{\mu 2}, L_{\mu 2}, C_{\mu 2}$ - respectively, resistances, capacitance and inductance of the magnetic circuit portions, respectively before and after the movable core;

$$
C_{\mu g}=\mu_{g} \mu_{0} \frac{S_{\mu}}{2 \delta} \text { - the capacity of the magnetic working }
$$
air clearance;

$\dot{I}_{\mu}=j \omega \dot{Q}_{\mu}$ - an integrated current of magnetic circuit.

Fig. 2. shows PSD of magnetic circuit made on the basis of the equivalent circuit,

To determine the analytical expression of magnetic currents $I_{\mu 1}$ and $I_{\mu 2}$ PSD - magnetic circuit for each elementary-level relationship between the magnetic current and voltage, and then by successive substitutions will find the desired current. Should begin with the value, which need to define, i.e.:

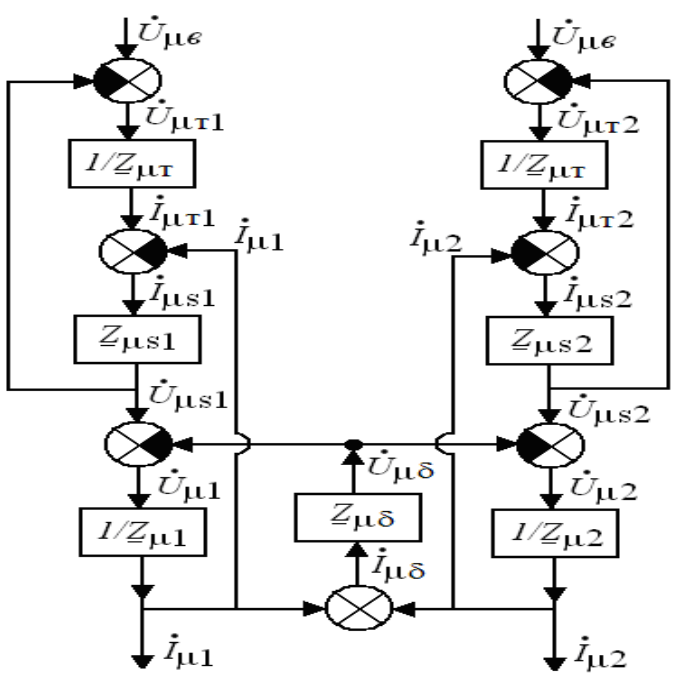

Figure 2. PSD of the magnetic circuit of electromagnetic transducers

$$
\begin{gathered}
\dot{I}_{\mu 1}=\frac{1}{\underline{Z}_{\mu 1}} \dot{U}_{\mu 1}=\frac{1}{\underline{Z}_{\mu 1}}\left(\dot{U}_{\mu S 1}-\dot{U}_{\mu \delta}\right), \\
\dot{U}_{\mu S 1}=\underline{Z}_{\mu S 1} \dot{I}_{\mu S 1}=\underline{Z}_{\mu S 1}\left(\dot{I}_{\mu \mathrm{r} 1}-\dot{I}_{\mu S 1}\right), \\
\dot{U}_{\mu \delta}=\underline{Z}_{\mu \delta} \dot{I}_{\mu \delta}=\underline{Z}_{\mu \delta}\left(\dot{I}_{\mu 1}+\dot{I}_{\mu 2}\right), \\
\dot{I}_{\mu T}=\frac{1}{\underline{Z}_{\mu \mathrm{T}}} \dot{U}_{\mu \mathrm{T} 1}=\frac{1}{\underline{Z}_{\mu \mathrm{T}}}\left(\dot{U}_{\mu \nu}-\dot{U}_{\mu S 1}\right), \\
\dot{I}_{\mu 2}=\frac{1}{\underline{Z}_{\mu 2}} \dot{U}_{\mu 2}=\frac{1}{\underline{Z}_{\mu 2}}\left(\dot{U}_{\mu S 2}-\dot{U}_{\mu \delta}\right), \\
\dot{U}_{\mu S 2}=\underline{Z}_{\mu S 2} \dot{I}_{\mu S 2}=\underline{Z}_{\mu S 2}\left(\dot{I}_{\mu \mathrm{T} 2}-\dot{I}_{\mu \delta}\right), \\
\dot{I}_{\mu \mathrm{T} 2}=\frac{1}{\underline{Z}_{\mu \mathrm{T}}} \dot{U}_{\mu \mathrm{T} 2}=\frac{1}{\underline{Z}_{\mu \mathrm{T}}}\left(\dot{U}_{\mu \nu}-\dot{U}_{\mu S 2}\right) .
\end{gathered}
$$

When substituting define and magnetic currents $I_{\mu 1}$ and $I_{\mu 2}$. At the same time, assume that $Z_{\mu \mathrm{T}}=0$.

During research of the electromagnetic transducer not considered an active and inductive resistance of the magnetic circuit, i.e. $R_{\mu}=0, X_{L \mu}=\omega L_{\mu}=0$. Expressions magnetic rigidity and magnetic containers for consideration of the magnetic circuit are as follows:

$$
W_{\mu 1}=\frac{\left(X_{\mathrm{M}}-x\right)\left[r^{2}+\left(R_{n}^{2}-R_{v}^{2}\right)\right]}{\mu \mu_{0} \pi\left(R_{n}^{2}-R_{v}^{2}\right) r^{2}}
$$

$W_{\mu 2}=\frac{\left(X_{\mathrm{M}}+x\right)\left[r^{2}+\left(R_{n}^{2}-R_{v}^{2}\right)\right]}{\mu \mu_{0} \pi\left(R_{n}^{2}-R_{v}^{2}\right) r^{2}}-$ magnetic inertial stiffening element of the cylindrical magnetic cores;

$$
\begin{gathered}
C_{\mu S 1}=\mu_{0} \frac{\pi\left(R_{v}+r\right)\left(X_{\mathrm{M}}-x\right)}{R_{v}-r} \\
C_{\mu S 2}=\mu_{0} \frac{\pi\left(R_{v}+r\right)\left(X_{\mathrm{M}}+x\right)}{R_{v}-r}-\text { magnetic cell capacity }
\end{gathered}
$$

of non-working parts of the air cleaners between cylindrical magnetic cores;

$$
W_{\mu \delta}=\frac{R_{v}-r-2 \delta}{\mu \mu_{0} \pi\left(R_{v}+r\right) b_{k}}+\frac{2 \delta\left(R_{v}+r\right)}{\mu_{g} \mu_{0} \pi\left(2 R_{v}-\delta\right)(2 r+\delta) b_{k}}
$$

- total magnetic rigidity of transmission cleaners, where $b_{k}$ - the width of the x-axis. 


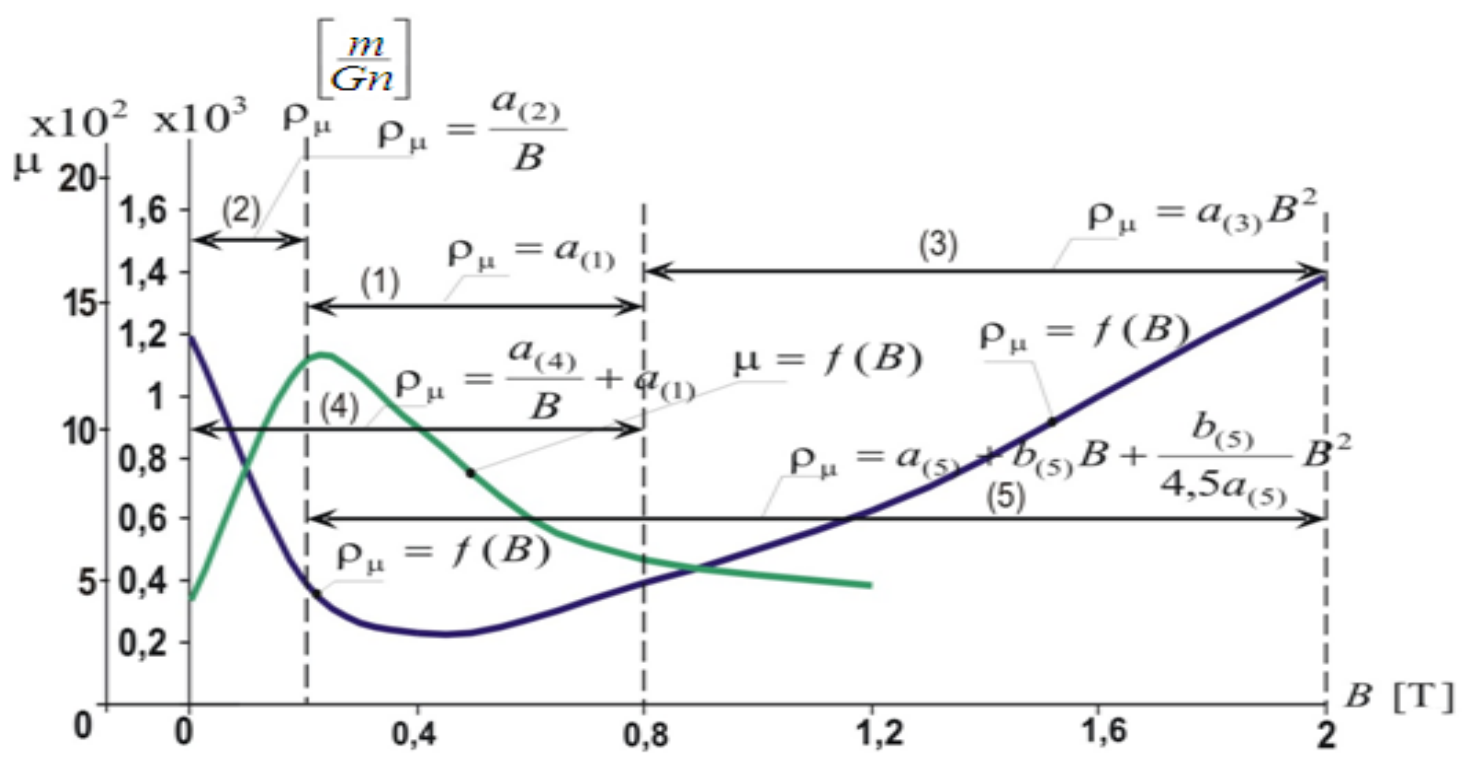

Figure 3. Curves and specific magnetic resistance of the magnetic permeability of steel from induction

\section{Nonlinear Dependence}

During research of electromagnetic converters used three-phases current ferromagnetic material, sometimes leads a sharp increase in labor induction in the chain to the point, where one can not neglect the nonlinear dependence $B=f(H)$. Accounting effect of non-linearity $B=f(H)$ of the dependence of effective value of magnetic flux from the voltage coordinates section under consideration using the curve $\rho_{\mathrm{M}}=f(B)$, where $\rho_{\mathrm{M}}$ - the specific resistance of the magnetic steel.

According to fig. 3 [3] depending on the range, which changes in the magnetic induction of the electromagnetic transducer section ferromagnet phase current, one can substitute a particular approximating function.

\section{Result \& Discussion}

In the article considered each of cases for determinate resistances of the electromagnetic transducer :

1. Induction of the magnetic steel in the cross section of the electromagnetic transducer phase current within a range of (1). This case is a most common in practice. In this case

$$
\rho_{\mu}=a_{(1)}
$$

2. Induction of the magnetic steel in cross section of the electromagnetic of three-phases $\mathrm{AC}$ inverter is changed within the range (2). In this case, the magnetic core is saturated and poorly fitting function can be written as

$$
\rho_{\mu}=\frac{a_{(2)}}{B} .
$$

3. Induction of sectional steel of electromagnetic transducer of phase of current varies within the range (3). In this range, the magnetic system is operating in a highly saturated state. The expression is inserted next approximating function:

$$
\rho_{\mu}=a_{(3)} B^{2} .
$$

4. Induction of the magnetic steel in cross section of the electromagnetic transducer phase current varies within a range (4). This range is typical for magnetic cores made of a material with a different non-linearity in the initial section. It is approximated by the dependence $\rho_{\mu}$ on the induction of the expression of this range

$$
\rho_{\mu}=a_{(4)}+b_{(3)} / B .
$$

5. Induction of the magnetic steel in the cross section of the electromagnetic transducer phase current varies within the range (5). It is quite rare for a range of the electromagnetic transducer of three-phase current, nevertheless represents a definite theoretical interest. The approximating function on this range was chosen mainly, because of the conditions of integrability of the resulting analytical differential equation has the form

$$
\rho_{\mu}=a_{(5)}+b_{(4)} B+\frac{b_{(4)}^{2}}{4,5 a_{(5)}} B^{2} .
$$

Fig. 4 shows the curve of change of magnetic flux for cases where the induction of the magnetic steel section in the electromagnetic transducer three-phase current changes within the allowable range.

The maximum value of the induction chosen equal $B=2,0 T$, which corresponds from magnetic flux $\dot{Q}_{\mu 0}=11,0 \cdot 10^{-5} \mathrm{~Wb}$. Other values used in the calculation are respectively: 


$$
\begin{aligned}
& S_{\mu}=6,5 \cdot 10^{-5} m^{2} ; C_{\mu r}=8,7 \cdot 10^{-6} \frac{G n}{m} ; W_{\mu \delta}=2 \cdot 10^{4} \frac{1}{G n} ; \\
& \underline{\gamma}_{\mathrm{cp}}=8,8 \frac{1}{G n} ; a_{(3)}=27 \frac{T}{G n} ; b_{(3)}=47 \cdot 10^{2} \frac{1}{T^{2} \cdot G n} .
\end{aligned}
$$

\section{Conclusions}

As shown from results of research of the influence of non-linearity of the magnetization curve for the primary flow distribution in a magnetic circuit of electromagnetic transducer of three-phases current ranges identified characteristic changes in the magnetic induction. As a result of research for each band proposed approximate functions as $\rho_{\mu}=f(B)$.

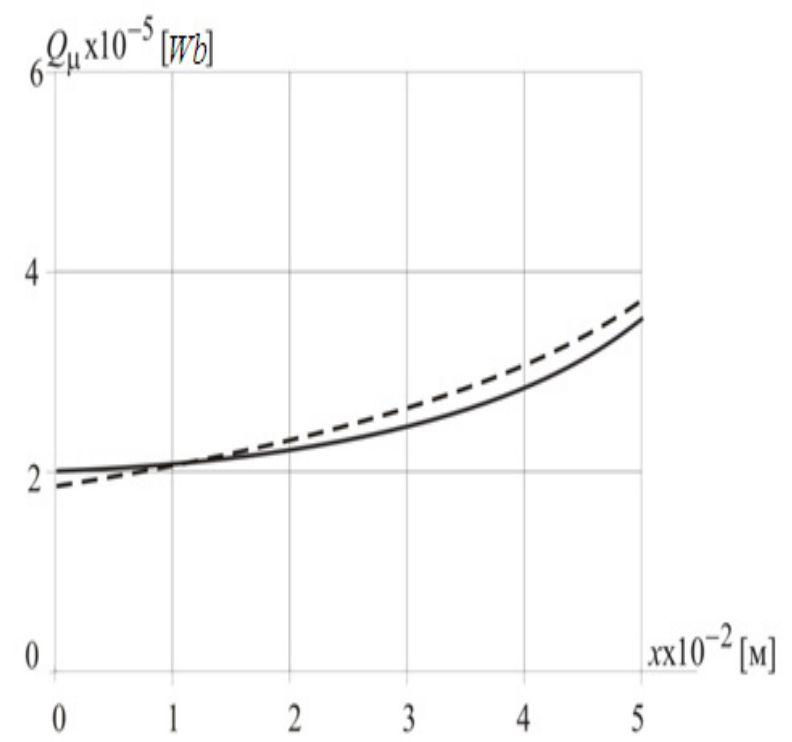

Figure 4. The curves of change of the magnetic flux along the magnetic circuit: solid line - estimated; the dotted line - pilot
As presented in fig. 4 the maximum difference between calculated and experimental dates equal 5.5\% [4]. Error of $5.5 \%$ it is defined on the basis of a difference between measured and theoretical characteristics.

\section{Acknowledgements}

The authors are deeply grateful for helps of Prof. Rakhmat Azimov and Ms. Dekhkanov Oybek during field studies.

We are also very grateful to experts for their appropriate and constructive suggestions to improve this template.

\section{REFERENCES}

[1] Siddikov I.Kh., Abdulaev A.Kh., Bobojanov M.K. Perfection and development of sensor controls and measuring transducers on a basis of information-energetics model. //WCIS - 2002. Collection of the works. II - World conf. 4-5 June 2002 y. b-Quadrat Verlag, Azerbaijan State Oil Academy, Azerbaijan, Baku, 2002. - p.p. 310 -315.

[2] Amirov S.F., Azimov R.K., Siddikov I.Kh., Khakimov M.Kh., Khushboqov B.X., Sattarov Kh.A. Patent RUz. №04185. The transducers of no symmetry of current to voltage.// Bulleten №6. 2010.

[3] Siddikov I.Kh. The Electromagnetic Transducers of Asymmetry of Three-phases Electrical Currents to Voltage. // Universal Journal of Electrical and Electronic Engineering. Horizon Research Publishing Corporation USA. 2015, Vol.3, N5, - p.146 - 148, http://www.hrpub.org.

[4] Siddikov I.Kh. Anarbaev M. A. Bedritskiy I. M., Khasanov M. Y. The analysis of base characteristics and inaccuracies of electromagnetic transducers current to voltage with flat measuring windings. // European Sciences review, Scientific journal, "East West" Association for Advanced Studies and Higher Education GmbH, Vienna, Austria, 2015, No 7-8, (July-August), p.137-139, http://www.ew-a.org. 